\title{
Correction to: \\ Impact of Mothers' Age on Telomere Length and Human Telomerase Reverse Transcriptase Expression in Human Fetal Membrane-Derived Mesenchymal Stem Cells by Alrefaei GI, Alkarim SA, and Abduljabbar HS. Stem Cells Dev 2019;28;24;1632-1645 DOI:10.1089/scd.2019-0144
} The authors of the article entitled, Impact of Mothers' Age on Telomere Length and Human Telomerase Reverse
Transcriptase Expression in Human Fetal Membrane-Derived Mesenchymal Stem Cells, published in Stem Cells Dev
2019;28;24:1632-1645 wish to correct errors that appeared in Figure 1 and Table 3.

In Figure 1, repetition occurred in day 9, where the hDB-MSCs - GiII ( $\geq 40$ years) was the same as hAM-MSCs - GII (30-39 years) and hCP-MSCs - GI (20-29 years) was the same as hUCWj - MSCs - GI (20-29 years).

The original figure is on the left. The authors have revised Figure 1 as shown on the right.

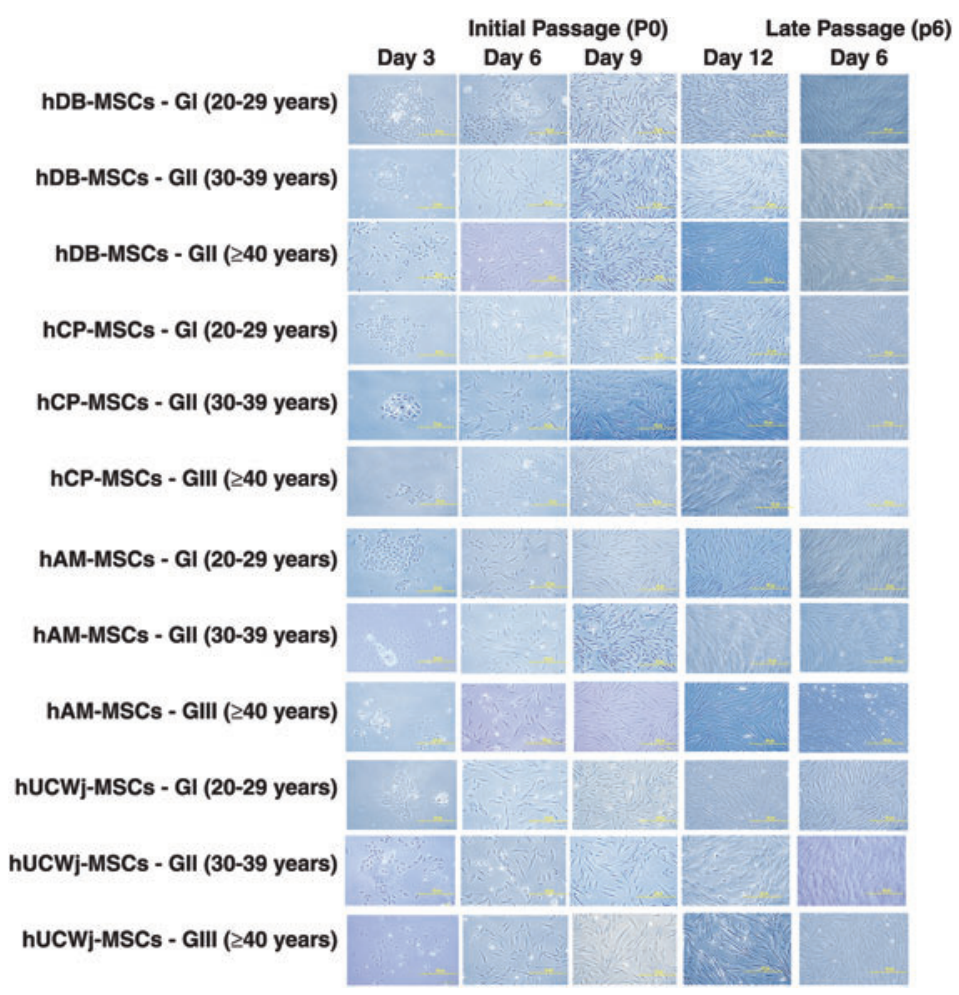

FIG. 1. Cellular morphology of hFM-MSCs. Representative images of hFM-MSCs [decidua basalis (hDB), chorionic plate (hCP), amniotic membrane (hAM), and umbilical cord Wharton's jelly (hWJSCs)] from the three different age groups during cell culture at the initial passage (P0) and late passage (P6). At day 3 and 6, hFMMSCs appear short, then from day 9 until 6th passages the cells appear fibroblast-like cells. They were longer, flattened, and have an expanded cytoplasm. (magnification $\times 10$ ). hFM-MSCs, human fetal membranes-mesenchymal stem cells. Color images are available online.

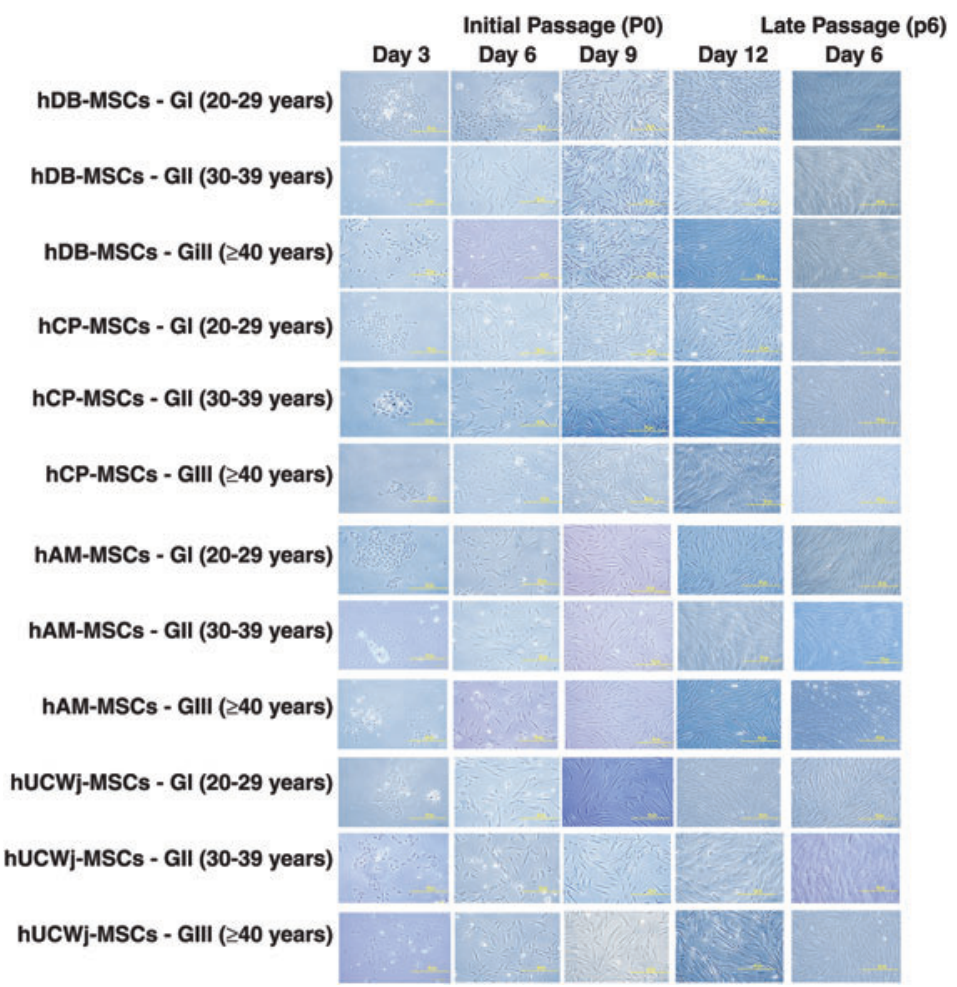

FIG. 1. Cellular morphology of hFM-MSCs. Representative images of hFM-MSCs [decidua basalis (hDB), chorionic plate (hCP), amniotic membrane (hAM), and umbilical cord Wharton's jelly (hWJSCs)] from the three different age groups during cell culture at the initial passage (P0) and late passage (P6). At day 3 and 6, hFMMSCs appear short, then from day 9 until 6th passages the cells appear fibroblast-like cells. They were longer, flattened, and have an expanded cytoplasm. (magnification $\times 10$ ). hFM-MSCs, human fetal membranes-mesenchymal stem cells. Color images are available online. 
In Table 3, the column hMSCs $\geq 40(\mathrm{n}=16)$, " $4 \mathrm{P}$ ' has been added to all groups.

The original Table 3 is on top and the revised Table 3 is shown on the bottom.

Table 3. Comparison of hFM-MSCs Proliferation Rates in Different Studied Groups by Doubling Time

\begin{tabular}{|c|c|c|c|c|c|c|c|c|}
\hline \multirow[b]{2}{*}{$\begin{array}{l}\text { Groups } \\
\text { Parameters }\end{array}$} & \multicolumn{3}{|c|}{ Control Samples } & \multirow{2}{*}{$\begin{array}{c}\text { hMSCs } \\
\text { 30-39 years } \\
(\mathrm{n}=16)\end{array}$} & \multirow{2}{*}{$\begin{array}{l}\text { hMSCs } \\
\geq 40 \text { years } \\
(\mathrm{n}=16)\end{array}$} & \multirow[b]{2}{*}{$\begin{array}{l}\text { Mean } \\
\text { square }\end{array}$} & \multirow[b]{2}{*}{ F-Factor } & \multirow[b]{2}{*}{ Significance } \\
\hline & $\begin{array}{l}\text { hMSCs 20-29 } \\
\text { years }(\mathrm{n}=16)\end{array}$ & $\begin{array}{c}K 562 \\
(\mathrm{n}=16)\end{array}$ & $\begin{array}{c}\text { hBM-MSCs } \\
(\mathrm{n}=16)\end{array}$ & & & & & \\
\hline $\begin{array}{l}\text { Decidua basalis }(\mathrm{hDB}) \\
\text { Significance }\end{array}$ & $45.90 \pm 0.01$ & $\begin{array}{l}19.39 \pm 0.31 \\
1 P<0.05\end{array}$ & $\begin{array}{c}75.42 \pm 0.04 \\
1 \mathrm{P}<0.05 \\
2 \mathrm{P}<0.05\end{array}$ & $\begin{array}{c}44.27 \pm 0.02 \\
1 \mathrm{P}<0.05 \\
2 \mathrm{P}<0.05 \\
3 \mathrm{P}<0.05\end{array}$ & $\begin{array}{c}43.98 \pm 0.01 \\
1 \mathrm{P}<0.05 \\
2 \mathrm{P}<0.05 \\
3 \mathrm{P}<0.05\end{array}$ & 1580.517 & 78295.110 & 0.001 \\
\hline $\begin{array}{l}\text { Chorionic plate }(\mathrm{hCP}) \\
\text { Significance }\end{array}$ & $46.59 \pm 0.02$ & $\begin{array}{c}19.39 \pm 0.31 \\
1 \mathrm{P}<0.05\end{array}$ & $\begin{array}{c}75.42 \pm 0.04 \\
1 \mathrm{P}<0.05 \\
2 \mathrm{P}<0.05\end{array}$ & $\begin{array}{c}45.15 \pm 0.04 \\
1 \mathrm{P}<0.05 \\
2 \mathrm{P}<0.05 \\
3 \mathrm{P}<0.05\end{array}$ & $\begin{array}{c}44.90 \pm 0.01 \\
1 \mathrm{P}<0.05 \\
2 \mathrm{P}<0.05 \\
3 \mathrm{P}<0.05\end{array}$ & 1575.452 & 76751.501 & 0.001 \\
\hline $\begin{array}{l}\text { Amniotic membrane (hAM) } \\
\text { Significance }\end{array}$ & $45.50 \pm 0.01$ & $\begin{array}{c}19.39 \pm 0.31 \\
1 \mathrm{P}<0.05\end{array}$ & $\begin{array}{c}75.42 \pm 0.04 \\
1 \mathrm{P}<0.05 \\
2 \mathrm{P}<0.05\end{array}$ & $\begin{array}{c}44.18 \pm 0.01 \\
1 \mathrm{P}<0.05 \\
2 \mathrm{P}<0.05 \\
3 \mathrm{P}<0.05\end{array}$ & $\begin{array}{c}43.93 \pm 0.02 \\
1 \mathrm{P}<0.05 \\
2 \mathrm{P}<0.05 \\
3 \mathrm{P}<0.05\end{array}$ & 1580.997 & 75142.437 & 0.001 \\
\hline $\begin{array}{l}\text { Umbilical cord Wharton's } \\
\text { jelly (hUCWJ) }\end{array}$ & $46.59 \pm 0.07$ & $19.39 \pm 0.31$ & $75.42 \pm 0.04$ & $45.22 \pm 0.03$ & $44.97 \pm 0.02$ & 1575.158 & 74510.786 & 0.001 \\
\hline Significance & & $1 \mathrm{P}<0.05$ & $\begin{array}{l}1 \mathrm{P}<0.05 \\
2 \mathrm{P}<0.05\end{array}$ & $\begin{array}{l}1 \mathrm{P}<0.05 \\
2 \mathrm{P}<0.05 \\
3 \mathrm{P}<0.05\end{array}$ & $\begin{array}{l}1 \mathrm{P}<0.05 \\
2 \mathrm{P}<0.05 \\
3 \mathrm{P}<0.05\end{array}$ & & & \\
\hline
\end{tabular}

Data are given as mean \pm standard deviation to compare hMSCs growth rates of hFM-MSCs (D) with different studied groups by doubling time (DT). Significance was determined using multivariate analysis and then significant data were analyzed by one-way analysis of variance (LSD) test. $P<0.05$ was considered significant. IP, significance versus hMSC from GI of the same region; $2 \mathrm{P}$, significance versus K562; 3P, significance versus hBM-MSC, 4P, significance versus hMSC from GII of the same region.

Table 3. Comparison of hFM-MSCs Proliferation Rates in Different Studied Groups by Doubling Time

\begin{tabular}{|c|c|c|c|c|c|c|c|c|}
\hline \multirow[b]{2}{*}{$\begin{array}{l}\text { Groups } \\
\text { Parameters }\end{array}$} & \multicolumn{3}{|c|}{ Control Samples } & \multirow{2}{*}{$\begin{array}{c}\text { hMSCs } \\
\text { 30-39 years } \\
(\mathrm{n}=16)\end{array}$} & \multirow{2}{*}{$\begin{array}{l}\text { hMSCs } \\
\geq 40 \text { years } \\
(\mathrm{n}=16)\end{array}$} & \multirow[b]{2}{*}{$\begin{array}{l}\text { Mean } \\
\text { square }\end{array}$} & \multirow[b]{2}{*}{ F-Factor } & \multirow[b]{2}{*}{ Significance } \\
\hline & $\begin{array}{l}\text { hMSCs } 20-29 \\
\text { years }(\mathrm{n}=16)\end{array}$ & $\begin{array}{c}K 562 \\
(\mathrm{n}=16)\end{array}$ & $\begin{array}{l}\text { hBM-MSCs } \\
\quad(\mathrm{n}=16)\end{array}$ & & & & & \\
\hline $\begin{array}{l}\text { Decidua basalis (hDB) } \\
\text { Significance }\end{array}$ & $45.90 \pm 0.01$ & $\begin{array}{c}19.39 \pm 0.31 \\
1 P<0.05\end{array}$ & $\begin{array}{c}75.42 \pm 0.04 \\
1 \mathrm{P}<0.05 \\
2 \mathrm{P}<0.05\end{array}$ & $\begin{array}{c}44.27 \pm 0.02 \\
1 \mathrm{P}<0.05 \\
2 \mathrm{P}<0.05 \\
3 \mathrm{P}<0.05\end{array}$ & $\begin{array}{c}43.98 \pm 0.01 \\
1 \mathrm{P}<0.05 \\
2 \mathrm{P}<0.05 \\
3 \mathrm{P}<0.05 \\
\mathbf{4 P}<\mathbf{0 . 0 5}\end{array}$ & 1580.517 & 78295.110 & 0.001 \\
\hline $\begin{array}{l}\text { Chorionic plate }(\mathrm{hCP}) \\
\text { Significance }\end{array}$ & $46.59 \pm 0.02$ & $\begin{array}{c}19.39 \pm 0.31 \\
1 \mathrm{P}<0.05\end{array}$ & $\begin{array}{c}75.42 \pm 0.04 \\
1 \mathrm{P}<0.05 \\
2 \mathrm{P}<0.05\end{array}$ & $\begin{array}{c}45.15 \pm 0.04 \\
1 \mathrm{P}<0.05 \\
2 \mathrm{P}<0.05 \\
3 \mathrm{P}<0.05\end{array}$ & $\begin{array}{c}44.90 \pm 0.01 \\
1 \mathrm{P}<0.05 \\
2 \mathrm{P}<0.05 \\
3 \mathrm{P}<0.05 \\
\mathbf{4 P}<\mathbf{0 . 0 5}\end{array}$ & 1575.452 & 76751.501 & 0.001 \\
\hline $\begin{array}{l}\text { Amniotic membrane (hAM) } \\
\text { Significance }\end{array}$ & $45.50 \pm 0.01$ & $\begin{array}{c}19.39 \pm 0.31 \\
1 \mathrm{P}<0.05\end{array}$ & $\begin{array}{c}75.42 \pm 0.04 \\
1 \mathrm{P}<0.05 \\
2 \mathrm{P}<0.05\end{array}$ & $\begin{array}{c}44.18 \pm 0.01 \\
1 \mathrm{P}<0.05 \\
2 \mathrm{P}<0.05 \\
3 \mathrm{P}<0.05\end{array}$ & $\begin{array}{c}43.93 \pm 0.02 \\
1 \mathrm{P}<0.05 \\
2 \mathrm{P}<0.05 \\
3 \mathrm{P}<0.05 \\
\mathbf{4 P}<\mathbf{0 . 0 5}\end{array}$ & 1580.997 & 75142.437 & 0.001 \\
\hline $\begin{array}{l}\text { Umbilical cord Wharton's } \\
\text { jelly (hUCWJ) }\end{array}$ & $46.59 \pm 0.07$ & $19.39 \pm 0.31$ & $75.42 \pm 0.04$ & $45.22 \pm 0.03$ & $44.97 \pm 0.02$ & 1575.158 & 74510.786 & 0.001 \\
\hline Significance & & $1 \mathrm{P}<0.05$ & $\begin{array}{l}1 \mathrm{P}<0.05 \\
2 \mathrm{P}<0.05\end{array}$ & $\begin{array}{l}1 \mathrm{P}<0.05 \\
2 \mathrm{P}<0.05 \\
3 \mathrm{P}<0.05\end{array}$ & $\begin{array}{l}1 \mathrm{P}<0.05 \\
2 \mathrm{P}<0.05 \\
3 \mathrm{P}<0.05 \\
\mathbf{4 P}<\mathbf{0 . 0 5}\end{array}$ & & & \\
\hline
\end{tabular}

Data are given as mean \pm standard deviation to compare hMSCs growth rates of hFM-MSCs (D) with different studied groups by doubling time (DT). Significance was determined using multivariate analysis and then significant data were analyzed by one-way analysis of variance (LSD) test. $P<0.05$ was considered significant. IP, significance versus hMSC from GI of the same region; $2 \mathrm{P}$, significance versus K562; 3P, significance versus hBM-MSC, 4P, significance versus hMSC from GII of the same region.

The online version of this article has been revised to reflect these corrections.

The authors sincerely regret these errors. For additional information, please contact: Dr. Ghadeer I. Alrefaei, Biology Department, Faculty of Sciences, University of Jeddah, Jeddah 22264, Saudi Arabia. E-mail: galrefaei@uj.edu.sa 